"This is the peer reviewed version of the following article: Martin SG. Spontaneous cell polarization: Feedback control of Cdc42 GTPase breaks cellular symmetry. 2015. Bioessays, which has been published in final form at DOI: 10.1002/bies.201500077. This article may be used for noncommercial purposes in accordance with Wiley Terms and Conditions for Self-Archiving."

\title{
Spontaneous cell polarization: Feedback control of Cdc42 GTPase breaks cellular symmetry
}

Sophie G. Martin

Department of Fundamental Microbiology, University of Lausanne, Switzerland

\section{Corresponding author:}

Sophie G. Martin

Sophie.Martin@unil.ch

Keywords: actin cytoskeleton; Cdc42 GTPase; feedback; Saccharomyces cerevisiae; Schizosaccharomyces pombe; spontaneous cell polarization; symmetry breaking

Abbreviations: GAP, GTPase activating protein; GDI, guanine nucleotide dissociation inhibitor; GEF, guanine nucleotide exchange factor.

\section{Summary}

Spontaneous polarization without spatial cues, or symmetry breaking, is a fundamental problem of spatial organization in biological systems. This question has been extensively studied using yeast models, which revealed the central role of the small GTPase switch Cdc42. Active Cdc42-GTP forms a coherent patch at the cell cortex, thought to result from amplification of a small initial stochastic inhomogeneity through positive feedback mechanisms, which induces cell polarization. Here, I review and discuss the mechanisms of Cdc42 activity selfamplification and dynamic turnover. A robust $\mathrm{Cdc} 42$ patch is formed through the combined effects of Cdc42 activity promoting its own activation and active Cdc42-GTP displaying reduced membrane detachment and lateral diffusion compared to inactive Cdc42-GDP. I argue the role of the actin cytoskeleton in symmetry breaking is not primarily to transport Cdc 42 to the active site. Finally, negative feedback and competition mechanisms serve to control the number of polarization sites. 


\section{Introduction}

Biological systems, from in vitro assemblies of few components to complex organisms, share the ability to self-organize, spontaneously breaking the symmetry of an initially homogeneous distribution. Spatial self-organization is particularly evident at the cellular level. Indeed, every cell within a multicellular organism, or any single-celled species, at least at some stage of its life cycle, displays a polarized organization necessary for its proliferation, differentiation or physiological function. While cells readily polarize in response to a spatial cue, they can also polarize in absence of spatial information [1]. This process, named 'spontaneous polarization' or 'symmetry-breaking', leads to polarization in random direction. Such phenomenon is observed ubiquitously, for instance in migratory cells, which migrate not only towards, but within homogeneous chemo-attractants [2]; in neurons, which extend neurites and specify an axon without spatial cues in vitro [3]; in plant cells to build a secondary cell wall [4]; or in simple unicellular yeasts, such as Saccharomyces cerevisiae and Schizosaccharomyces pombe, which exhibit spontaneous polarization upon spore germination or when the historical landmarks that normally orient growth are absent. Extensive work in organisms ranging from prokaryotes to animals has revealed the central role of small GTPases in this process. In this review, I focus on recent studies of the highly conserved Cdc42 GTPase in yeasts, and discuss the molecular mechanisms by which this small GTPase breaks symmetry.

Cdc42 is a member of the eukaryotic Ras-homologous Rho-family of GTPases, itself part of the wider super-family of small GTPases, including Rop GTPases in plants and small GTPases in prokaryotes, many of which are involved in cell polarization [5-7]. Like all of these GTPases, Cdc42 acts as a switch (Figure 1): It is active when loaded with GTP; its GTPase activity, with the help of GTPase activating proteins (GAPs), hydrolyses GTP to GDP, converting it to the inactive form. Re-activation occurs upon exchange of GDP with a new GTP molecule, a process catalysed by guanine nucleotide exchange factors (GEFs). Cdc42, like most small eukaryotic GTPases, is membrane associated due to cysteine prenylation of its C-terminal CAAX box. Insertion of newly prenylated proteins is thought to take place in ER membranes and is followed by trafficking through the endomembrane system to the plasma membrane [8,9]. Cdc42 can also be maintained in the cytosol upon binding to guanine nucleotide dissociation inhibitors (GDIs), which shield the C-terminal end of the molecule including the prenyl group to solubilize the protein [10].

The evidence that Cdc42 is able to impel symmetry breaking in absence of an upstream spatial signal comes from groundbreaking work, presented below, in yeast. Both Cdc42 activity and protein levels polarize to a single site at presumptive sites of growth in otherwise spherical cells, forming a 'Cdc42 patch' or 'polar cap'. Local Cdc42 activity then promotes the activation of a host of effectors, including PAK-family kinases, organizers of the actin cytoskeleton and organizers of vesicle exocytosis [11-14]. In yeast cells in particular, Cdc42 activity leads to activation of formins [15-18], which assemble actin tracks for polarized vesicle transport, and activation of the exocyst [19-22], a multisubunit complex that tethers incoming vesicles at the plasma membrane for secretion. These pathways collectively transduce the initial polarization of Cdc42 activity into effective cytoskeletal and cell polarization and the emergence of a new 
growth site. Thus a central question is: "What self-organization principles underlie the formation of a robust patch of Cdc42 activity?"

Conceptually, the answer is simple: a positive feedback mechanism serves to amplify a small initial inhomogeneity in Cdc42 localization/activity for formation of a coherent patch. This simple concept is supported by a large body of theoretical work first inspired by Turing reaction-diffusion systems [23]. Two prominent positive feedback mechanisms have been proposed - that Cdc42 activity promotes its own activation through a reaction-diffusion system [24,25], and that Cdc42 promotes its own local enrichment through an actin-based transport system [26]. Over the past 10 years, these two ideas have often been considered in opposition or as distinct mechanisms collectively providing robustness to cell polarization establishment. In this manuscript, I aim to go beyond this dichotomy and present a critical view of our current understanding of Cdc42 spontaneous polarization, including less discussed aspects of Cdc42 regulation. In my discussion, I strive to use precise terminology to distinguish mechanisms acting on Cdc42 activation (i.e. its GTP-GDP cycle) from those acting on Cdc42 protein localization, which can act differentially on the active and inactive form. I do not discuss in detail the large body of theoretical models that helped nourish the field, and which have been recently reviewed [27]. Instead I chose to focus on the experimental dissection of Cdc42 regulation, also discussing additional principles that are required together with positive feedbacks to control the size and number of Cdc42 patches.

\section{Self-amplification of Cdc42 activity for symmetry breaking}

\section{The Bem1-GEF-PAK complex}

The local self-amplification of Cdc42 activity can be achieved through a positive feedback mechanism in which active Cdc42 promotes the activation of neighbouring Cdc42 molecules. The best understood molecular mechanism underlying such positive feedback involves the formation of a protein complex between a Cdc42 effector, a PAK-family kinase, which binds Cdc42-GTP, and a Cdc42 GEF, which activates Cdc42 [25,28,29]. This complex forms through a protein, named Bem 1 in $S$. cerevisiae, which directly binds both the PAK and the GEF, as well as Cdc42-GTP itself (Figure 2). I note that although Bem1 is often referred to as a scaffold, this should not be understood to mean that the protein is stably anchored at the polarity site, as it exhibits fast dynamics similar to Cdc42 (see below) [30]. A similar complex was also described both in vivo and in vitro in the fission yeast $[31,32]$, suggesting this positive feedback regulation may be conserved, though the possible role of this complex in spontaneous polarization in fission yeast has not been tested.

The experimental evidence that this complex underlies feedback enhancement of Cdc42 activity stems from synthetic and loss-of-function approaches. Indeed, the artificial formation of a GEF-PAK complex in absence of Bem1 is sufficient to promote the formation of a site of polarity in conditions when Bem 1 is essential, and disruption of the complex abrogated spontaneous polarization in these same conditions [29]. Although structural and biochemical information has begun to reveal the inner workings of this complex, many details remain unknown. For instance, is there a specific requirement for the PAK kinase activity, or can the 
mere physical linkage of a GEF to Cdc42-GTP mediate the positive feedback? Phosphorylation of the GEF by the PAK is prominent [33], but analysis of the consequence of this phosphorylation has yielded conflicting results [34,35], with recent data suggesting a negative influence of phosphorylation on GEF activity [36]. Bem 1 also binds other Cdc42 effectors, including two subunits of the exocyst, an octameric complex that tethers secretory vesicles at the plasma membrane for fusion $[37,38]$. Interestingly, this interaction is compatible with Bem1 also binding the Cdc42 GEF such that a ternary complex can form at least in vitro [38]. This raises the question of whether Bem 1 may link the Cdc42 GEF with several Cdc42 effectors to promote feedback activation of Cdc42.

\section{A Ras-mediated positive feedback?}

The Bem 1 complex is not the only means of local amplification of Cdc42 activity. While the GEF Cdc24 is essential for viability, Bem1's essential role in spontaneous polarization is only revealed in mutant backgrounds lacking the Ras-like small GTPase Rsr1. The canonical view of cell polarization in yeast, built upon decades of work, is a three-layered system $[39,40]$. The first consists of landmarks providing spatial information. In turn, these recruit the central polarization machinery built around Cdc42. Finally, Cdc42 activates effectors to polarize secretion for effective cell polarization. As cells lacking Rsr1 display efficient budding but do so at random location [41], and landmark signals converge onto Rsr1 activation [42], rsr1 $1 \Delta$ backgrounds have been used as a convenient means to remove landmark information and study spontaneous polarization. However, in mutants lacking transmembrane landmark proteins, which bud at random location but express Rsr1, the Bem1-dependent positive feedback described above is not essential $[25,43]$. One conclusion is that Rsr1, in addition to its function in linking Cdc42 to the spatial landmarks, must also contribute to the spontaneous, landmark-independent, polarization of Cdc42 (Figure 2).

A large body of work has shown that Rsr1 activates Cdc42. Like Bem1, Rsr1-GTP binds the Cdc42 GEF Cdc24 and promotes its release from auto-inhibition [4446]. As Cdc24 is essential, this shared role of Rsr1 and Bem1 in Cdc24 activation may indeed underlie their synthetic lethality [47]. Active Rsr1 also directly binds inactive Cdc42 and this interaction is reinforced by Cdc24 [48,49], and all three proteins can likely form a complex as Cdc24 reinforces the Rsr1-Cdc42 interaction, perhaps by inhibiting Rsr1 GTPase activity $[46,48]$. Interestingly, Rsr1 also directly binds Bem1 [45], suggesting strong inter-connection with the Bem1-GEF-PAK feedback described above. Data from the fission yeast model is also suggestive of a role for Ras1 (the only Ras GTPase in this organism) in Cdc42 activation, through interaction with a Cdc42 GEF [32]. A major question for the future is whether and how Cdc42 activity may positively feedback onto Ras activation for spontaneous polarization.

\section{Symmetry-breaking through a second Cdc42 GEF}

Cells may possess additional mechanisms to those described above for Cdc42 activity self-amplification. This is best illustrated in the fission yeast, where two 
distinct GEFs, Scd1 and Gef1, activate Cdc42. Scd1 is the S. pombe Cdc24 homolog, and may as in $S$. cerevisiae be modulated by the established Bem1/Scd2-dependent and putative Rsr1/Ras1-dependent positive feedbacks $[31,32]$. However, deletion of any of these genes is not lethal in fission yeast, and round spores or spheroplasts are able to break symmetry and polarize a single, albeit wider, growth zone, yielding very fat cells [50,51]. The regulatory controls behind the second Cdc42 GEF, Gef1, which does not have a direct homolog in $S$. cerevisiae, are still poorly understood, though its localization at cell poles is influenced by both a NDR kinase and microtubule-delivered landmarks $[52,53]$. Interestingly, Gef1 does not strongly influence cell morphology, but is required for bipolarity and may allow activation of Cdc42 on cell sides upon stress $[54,55]$. As Gef1 is essential in the absence of Scd1 [55,56], one conclusion is that this second GEF can also promote apparent spontaneous polarization (Figure 2).

A second Cdc42 GEF, named Bud3, best known for its role in the axial landmark [42], was also very recently described in S. cerevisiae [57]. Interestingly, Bud3 activity is required for the formation of stable complexes between the Rsr1 GEF and the landmark, suggesting a possible feedback circuit of Cdc42 activation on Rsr1. Both Bud3 and Rsr1 are required for a first phase of Cdc42 polarization in the early G1 phase when Cdc24 is held in the nucleus $[57,58]$. This early polarization is not normally sufficient for bud formation, because the main Cdc42 GEF Cdc24 is essential for life. Whether Bud3 can sustain symmetry breaking or only functions with the landmark is not known. However, as the bem1 $1 \Delta$ rsr1 1 double mutant is viable in some strain backgrounds [43], and a hyperactive $c d c 42$ mutant allele can live without Cdc24 [59], the cell may have back-up systems to break symmetry.

In conclusion, cells likely possess highly inter-connected circuits in addition to the often-discussed Bem1-GEF-PAK feedback for the local feedback amplification of Cdc42 activity.

\section{Local Cdc42 enrichment at sites of activity}

Cdc42 protein is enriched at the polar cap and the local amount of Cdc42 protein strongly correlates with its local activity level [60]. This local protein accumulation requires dynamics of Cdc42 protein. Indeed, Cdc42, like other components of the polarity patch, is highly dynamic, exhibiting exchange halftimes of a few seconds. Three main modes have been discussed to underlie this dynamic exchange of Cdc42: 1) extraction from and insertion into the plasma membrane, mediated by the GDI; 2) lateral diffusion within the plasma membrane; and 3) actin-dependent delivery and internalization of Cdc42 on exoand endocytic vesicles. Each of these modes contributes to Cdc42 dynamics at distinct time scales, from the fastest GDI-mediated membrane-cytosol exchange occurring within seconds to the slowest actin-dependent vesicular transport (within tens of seconds to minutes).

\section{Extraction from the membrane}

Rho GDIs maintain Rho GTPases soluble in the cytosol by shielding the membrane-targeting lipid modification at their C-terminus. Though GDIs can 
interact with both GTP- and GDP-loaded Rho GTPases, it has been postulated that extraction from the membrane is initiated by GTP hydrolysis [10], and thus the GDI specifically extracts Cdc42-GDP from the membrane (Figure 3). Inclusion of this simple idea in a theoretical model of positive feedback regulation of Cdc42 activity is sufficient to yield spontaneous enrichment of Cdc42 at the site of activity [24]. Because of proposed competition between GEF and GDI for Rho binding, a positive feedback for Cdc42 recruitment to the membrane has been suggested, in which Cdc42 is preferentially released from the GDI and inserted into the membrane at the site of GEF accumulation [61]. Interestingly, deletion of the sole GDI Rdi1 in yeast, which extracts Cdc42 but also other Rho-family GTPases from the membrane [62,63], slows down Cdc42 dynamics (about 4-fold in $S$. cerevisiae [64] and less than 2 -fold in $S$. pombe [60]), but does not prevent Cdc42 polarization, indicating that other modes of Cdc42 dynamics also contribute.

\section{Trafficking of Cdc42 on vesicles}

The contribution of the actin-dependent delivery and internalization of Cdc42 on exo- and endocytic vesicles to spontaneous polarization has nourished a heated debate for several years now. Early attention to this dynamic mechanism was brought about by the compelling demonstration of a positive feedback involving Cdc42 transport on vesicles in S. cerevisiae: overexpression of a constitutively active Cdc42 allele in unpolarized G1-arrested cells is sufficient to drive the formation of polar caps [26]. The positive feedback stems from the fact that Cdc42 activity underlies actin cable assembly and polarized secretion, which are required for this spontaneous polarization. The observations that $\mathrm{Cdc} 42$ is present on exocytic vesicles and that even an inactive Cdc42 allele co-expressed in wild type cells accumulates at the polarity patch support the idea that local Cdc42 activity promotes the delivery of additional Cdc42 molecules [26,30,65].

The debated question has been to what extent this positive feedback contributes to polarization in cells with wildtype Cdc42. Several studies suggested that actinbased trafficking of Cdc42 and GDI-mediated membrane-cytosol shuttling are together essential for Cdc42 polarization, because co-disruption of polarized secretion and the GDI prevents Cdc42 polarization [61,64]. However, arguments have been made to suggest that trafficking of Cdc42 does not strongly contribute to spontaneous polarization. The first concerns the timescale: in the artificial system, constitutively active Cdc42 exhibits much slower exchange dynamics (with minute halftime) than wild type Cdc42 (seconds), and several minutes are required for it to polarize $[26,30]$. Thus, this raises questions about the contributions of this slower mechanism, especially considering that only a small fraction of Cdc42 is in the active state in wild type cells. Polarized actin is also observed only a few minutes after the first apparent Cdc42 polarization [66]. Second, quantitative and theoretical arguments have been made around the relatively lower concentration of Cdc42 on vesicles than at the polarized patch and on the contribution of membrane flux, which may dilute rather than concentrate Cdc42 at the plasma membrane [67-69]. Some of these arguments have recently been countered by showing that Cdc42 forms micro domains at the membrane with distinct diffusion rates, which permit a positive effect of Cdc42 
delivery for polarization [70]. Finally, we recently reported an engineered Cdc42 allele that does not visibly associate with exocytic vesicles, yet is able to efficiently polarize spontaneously in both $S$. pombe and $S$. cerevisiae [60]. In this allele, the Cdc42 C-terminal CAAX box, which is normally prenylated in the cytosol for membrane insertion at the ER, is replaced by an amphipathic helix, which can associate with the plasma membrane directly without transit through the secretory system. Because the prenyl moiety is absent in this allele, it is also unlikely to be a substrate for the GDI, suggesting efficient local enrichment of Cdc42 even in the absence of both modes of dynamic exchange. Thus, my take from all these studies is that, although actin-based vesicle trafficking plays an important role in polarization (see below), the key cargo is unlikely to be Cdc42.

\section{Lateral diffusion of Cdc42}

In the absence of GDI-mediated membrane-cytosol shuttling and vesiclemediated recycling of $\mathrm{Cdc} 42$, what then promotes Cdc42 dynamics for local enrichment? Lateral diffusion is one possible factor. This is generally described as a negative factor that acts against local protein concentration. For instance, transmembrane proteins that diffuse very slowly at the yeast plasma membrane (with a diffusion rate of $\sim 0.0025 \mu \mathrm{m}^{2} / \mathrm{s}$ ) can be dynamically polarized by endocytic recycling retrieving diffusing proteins and re-inserting them at the centre of the polarized patch [71], but fast diffusion overcomes this effect. The diffusion of GFP-tagged Cdc42 in S. cerevisiae has been measured at rates ranging between $\sim 0.0061$ and $\sim 0.053 \mu \mathrm{m}^{2} / \mathrm{s}[70,72]$, slow enough to allow polarization [72]. One important caveat to be noted is that these measurements used GFPtagged Cdc42, which, by many criteria, is only partly functional $[61,69,73]$. Recent measurements of Cdc42 diffusion in $S$. pombe, using a functional Cdc42fluorescent fusion, revealed much faster diffusion, up to $\sim 0.2 \mu \mathrm{m}^{2} / \mathrm{s}$ [60]. It will be important to clarify whether these significant differences in diffusion rates are inherent to the distinct organisms under study or reflect differences in Cdc42 labelling.

In either case, if Cdc42 diffuses fast, how can a local zone of enrichment be formed and maintained? The key answer to this question likely lies in the observation that Cdc42 diffusion rates are non-uniform: Cdc42 diffuses very fast outside of polar caps (at $\sim 0.2 \mu \mathrm{m}^{2} / \mathrm{s}$ ), but significantly slower at polar caps. A strong correlation between the local levels of active Cdc42 and its diffusion rate further suggests that active Cdc42 diffuses slower than the inactive form. The precise diffusion rate of Cdc42-GTP could thus far not be measured, but was estimated to be at least one order of magnitude slower than that of Cdc42-GDP. This observation leads to the idea that the fast diffusion of Cdc42-GDP can fuel the growth of relatively stable Cdc42-GTP patches, and thus act to reinforce polarization rather than disperse it (Figure 3). As Cdc42-GDP is consumed by conversion into the more stable Cdc42-GTP at the polar cap, there is a larger influx than loss of Cdc42 as the cap is growing. This idea has not been stringently examined from a theoretical standpoint, but can essentially be considered as Turing-type mechanism [24], reduced to two dimensions.

In summary, the enrichment of Cdc42 at sites of activity may simply result from the comparatively slower dynamics of the active form, both for membrane 
extraction and along the plane of the membrane. Whether this accumulation participates in symmetry breaking, or is a simple consequence of local activation is an open question. Though a Cdc42 allele stably tethered by a transmembrane domain to the plasma membrane in fission yeast displays polarized activity without local accumulation, this allele is clearly hypomorphic [60]. What mediates the differential turnover of active and inactive Cdc42 is also an important future question. One contribution may come from the lipid microenvironment at the plasma membrane. For instance, the negatively charged phosphatidyl-serine lipid is polarized at the membrane, co-enriched with Cdc42 in micro domains and required for Cdc42 local enrichment [70,74], possibly by restricting Cdc42 diffusion as well as its extraction by the GDI [75]. Whether such micro domains may be triggered by Cdc42 activity is an open question.

The actin cytoskeleton-based vesicular trafficking reinforces spontaneous polarization

Though actin-based vesicular transport of Cdc42 may not be as important as initially thought, the contribution of actin-based vesicular transport to spontaneous polarization is well documented (Figure 4). Actin depolymerization destabilizes the polarized patch in both $S$. cerevisiae and $S$. pombe $[22,30]$. In the former case, actin depolymerization promotes patch instability [30,73]. Concomitant actin disruption with deletion of bem1 (blocking the GEF-PAK positive feedback on Cdc42 activation) or rdi1 (abrogating Cdc42 membrane detachment) abolishes Cdc42 local enrichment [30,61,64]. Remarkably, polarization driven by an artificial GEF-PAK complex also relies on F-actin [43]. In $S$. pombe, sustained actin depolymerization leads to the formation of dynamic Cdc42 patches, which form and disassemble around the cell cortex, even upon deletion of rdi1 [22,60]. This actin disruption-induced dynamics is also observed with the Cdc42 allele that does not bind vesicles, indicating that the actin cytoskeleton impinges on a target(s) other than Cdc42 itself [60].

The actin cytoskeleton may reinforce polarization through diverse actions, which may be distinct in the two species. A recent study suggested a role for the septin cytoskeleton in Cdc42 patch destabilization in S. cerevisiae [76]: Cdc42-GTP recruits the septins, which provide a negative feedback by associating with a Cdc42 GAP. In the absence of F-actin and polarized exocytosis, this leads to an oscillatory dynamics of Cdc42 patch assembly and destabilization. In normal conditions, the polarized delivery of exocytic vesicles along actin cables sculpts a hole within the septin patch, thus displacing the septins and the negative feedback signal to a peripheral ring, which then delimits the mother cell from the bud. In this scenario, vesicular transport acts to displace a negative regulator of Cdc42.

The actin cytoskeleton also likely serves to counteract the disruptive effects of membrane flux. I noted above how actin-directed membrane trafficking may dilute Cdc42 or regulators $[67,68]$. In one beneficial example of this phenomenon, polar cap destabilization allows cells to better orient along a chemotropic gradient [77]. Actin-driven endocytosis also antagonizes the polar cap. This effect was revealed upon disruption of polarized exocytosis when the 
balance between influx and recycling is disrupted $[65,78]$. Thus, actin-dependent polarized transport plays a globally positive role counteracting the effect of membrane flux. One possibility is that the actin cytoskeleton acts positively on an upstream regulator of $\mathrm{Cdc} 42$, similar to what has been proposed for significantly faster growing pollen tubes [79].

\section{Polarized zones are destabilized by negative feedbacks and competition}

Positive feedbacks are not sufficient to generate a single, in case of budding yeast, or two stable polar caps, in case of fission yeast, of defined, restricted size. Additional regulatory mechanisms, recently reviewed elsewhere [80,81], include: the limiting nature of one or several factors, preventing the positive feedbacks to activate the entire cell cortex [82]; competition over these limiting factors between concurrently emerging polar caps for resolution, in $S$. cerevisiae, to a single one [73], and, in S. pombe, to two oscillating ones [83]; and negative feedbacks, which facilitate this competition.

The evidence for negative feedbacks stems from observations of wave-like and oscillatory dynamics, indicating reversal of polar cap growth by a negative control $[73,83,84]$. This is perhaps best exemplified in $S$. pombe, where Cdc42 activity exhibits oscillations between the two cell poles during bi-polar cell growth [83], around the cell cortex in mating cells exposed to low levels of pheromone [85], and around the spore periphery before outgrowth [86]. Negative feedbacks have also been described in $S$. cerevisiae, with multiple zones of Cdc42 activity transiently seen to emerge and oscillate before stabilization of a single one in $r s r 1 \Delta$ [73], and also in early G1 wildtype cells [58,87], but another study did not observe transient multisite polarity emergence [88]. In either system, the mechanisms underlying the negative feedback and its observed delay over polar cap formation are not yet well understood. GEF phosphorylation by the PAK kinase may play an inhibitory feedback function [36,83]. As mentioned above, septins also contribute to negative feedback signalling to destabilize the Cdc42 patch by recruiting Cdc42 GAPs to the polar cap [76]. Cdc42 GAPs are well established to limit Cdc42 activity, for instance by constraining Cdc42 activity to a limited area restricting the width of the fission yeast cell $[89,90]$, or by preventing Cdc42 activity at the previous division scar in the budding yeast cell [91]. They are thus prime candidates for negative feedback signalling, though evidence of feedback control is currently lacking. Finally, a beautiful recent study showed that the physical properties of the spore wall, a rigid protective shell surrounding spores, contribute to the destabilization of polarity patches before spore outgrowth, leading to an average isotropic growth that stretches the spore wall, before local breakage of the spore wall stabilizes the polarity patch [86].

One puzzling question is what defines the number of polar caps, and thus sites of growth. The optimal number of sites is different in different organisms. In $S$. cerevisiae, singularity is of the essence, because the bud neck defines the future site of cell division, and thus a single site is required for equal repartition of the genetic material at division [61]. One idea is that emerging caps are in competition for a limiting component, which is eventually taken up by a single cap, ensuring singularity. However, both the substrate and the nature of the competition are poorly defined. Several mutant conditions yield concurrent 
stable polar caps, even leading to budding at two sites. These include notably an hyperactive $c d c 42$ allele that bypasses GEF requirement [59]; an engineered cdc42 allele targeted to the membrane by an amphipathic helix [60]; deletion of Cdc42 GAPs [92]; deletion of the GDI [88]; and a strain in which a positive feedback is engineered through actin-based delivery of Bem1 [66]. One common point between the first four conditions is that Cdc42 turnover is dampened, either by directly interfering with its dynamics, or indirectly because slower GTP hydrolysis may stabilize Cdc42 association with the membrane. In the last situation, it is the turnover of Bem 1 that is strongly reduced. Thus, fast exchange dynamics of Cdc42 and other polar cap components favour competition, with the slower exchanging one likely limiting the competition.

What then allows multiple sites to co-exist, such as in the fission yeast, which grows in a bipolar manner through most of its cell cycle? One first idea is that bipolarity results from saturation at the first polarized zone, allowing initiation of a second one [83]. A second idea is that bipolarity could be achieved by tuning the exchange dynamics of Cdc42 and regulators. Indeed, the handful of conditions that cause premature bipolarity in the fission yeast includes some of the same mutants described in $S$. cerevisiae to cause multiple concurrent polar caps, namely $c d c 42$ alleles with altered modes of membrane targeting [60], deletion of a Cdc42 GAP [89], as well as alterations in the actin cytoskeleton $[93,94]$. However, there is no evidence of natural changes in exchange dynamics when cells shift from their mono- to bipolar mode of growth. Interestingly, in several of the large number of mutant situations that cause failure in bipolarity [95], the amount of active Cdc42 at the single growing end is significantly higher than at each end of a wild type bipolar cell $[60,83]$. This suggests that, for bipolarity, competition may favour re-distribution between sites, rather than resolution to a single site. I note that bipolarity in $S$. pombe also requires a set of landmark factors, normally deposited at cell poles by the microtubule cytoskeleton [96-98]. These factors also have the ability to form additional polarity zones when they are artificially targeted to cell sides $[53,99,100]$. They may define preferred regions for polar cap assembly by somehow biasing the local activation of $\mathrm{Cdc} 42$, though the precise mechanisms remain to be uncovered. Future studies should determine how limitation and dynamic turnover are tailored to achieve distinct numbers of polarization sites.

\section{Conclusions}

In conclusion, cells evolved highly robust symmetry-breaking mechanisms that rely on intertwined feedback amplification of local Cdc42 activity. In turn, local Cdc42 activity favours Cdc42 accumulation because active Cdc42 is poorly mobile relative to the inactive form. How these feedbacks are modulated to give rise to single or double sites of growth remains however poorly understood. In this respect, dynamic turnover of $\mathrm{Cdc} 42$ and its regulators, and their possible mobilization through negative feedback, are critical ingredients for competition to define the appropriate number of sites of polarity. These mechanisms may well apply beyond yeast cells to other Rho or Ras-family GTPases, with Cdc42 merely representing a case-in-point study for the spatial organization of this important family of regulators. 
The highly complex situation in vivo renders it difficult to dissect even with careful top-down approaches, considering every bilateral interaction. While this has been complemented by synthetic approaches reconstructing feedback mechanisms or Cdc42 membrane binding through artificial, informative linkages, in the future, bottom-up approaches, either in vitro or in heterologous systems, may offer complementary views on the basic build up of spontaneous polarization.

\section{Acknowledgments}

I am grateful to Laura Merlini, Serge Pelet and Aleksandar Vjestica for discussions and critical reading of the manuscript. Work in my lab is supported by a Swiss National Science Foundation Grant (31003A_155944) and an ERC Starting Grant (GeometryCellCycle).

\section{References}

1 Wedlich-Soldner R, Li R. 2003. Spontaneous cell polarization: undermining determinism. Nat Cell Biol 5: 267-70.

2 Petrie RJ, Doyle AD, Yamada KM. 2009. Random versus directionally persistent cell migration. Nat Rev Mol Cell Biol 10: 538-49.

3 Dotti CG, Sullivan CA, Banker GA. 1988. The establishment of polarity by hippocampal neurons in culture. J Neurosci 8: 1454-68.

4 Oda Y, Fukuda H. 2014. Emerging roles of small GTPases in secondary cell wall development. Frontiers in plant science 5: 428.

5 Yang Z, Lavagi I. 2012. Spatial control of plasma membrane domains: ROP GTPase-based symmetry breaking. Current opinion in plant biology 15: 601-7.

6 Bulyha I, Hot E, Huntley S, Sogaard-Andersen L. 2011. GTPases in bacterial cell polarity and signalling. Curr Opin Microbiol 14: 726-33.

7 Gonzalez-Billault C, Munoz-Llancao P, Henriquez DR, Wojnacki J, et al. 2012. The role of small GTPases in neuronal morphogenesis and polarity. Cytoskeleton (Hoboken) 69: 464-85.

8 Choy E, Chiu VK, Silletti J, Feoktistov M, et al. 1999. Endomembrane trafficking of ras: the CAAX motif targets proteins to the ER and Golgi. Cell 98: 69-80.

9 Wright LP, Philips MR. 2006. Thematic review series: lipid posttranslational modifications. CAAX modification and membrane targeting of Ras. Journal of lipid research 47: 883-91.

10 DerMardirossian C, Bokoch GM. 2005. GDIs: central regulatory molecules in Rho GTPase activation. Trends Cell Biol 15: 356-63.

11 Rincon SA, Estravis M, Perez P. 2014. Cdc42 regulates polarized growth and cell integrity in fission yeast. Biochem Soc Trans 42: 201-5.

12 Harris KP, Tepass U. 2010. Cdc42 and vesicle trafficking in polarized cells. Traffic 11: 1272-9.

13 Melendez J, Grogg M, Zheng Y. 2011. Signaling role of Cdc42 in regulating mammalian physiology.J Biol Chem 286: 2375-81. 
14 Martin SG, Arkowitz RA. 2014. Cell polarization in budding and fission yeasts. FEMS microbiology reviews 38: 228-53.

15 Chen H, Kuo CC, Kang H, Howell AS, et al. 2012. Cdc42p regulation of the yeast formin Bni1p mediated by the effector Gic2p. Mol Biol Cell 23: 3814-26.

16 Dong Y, Pruyne D, Bretscher A. 2003. Formin-dependent actin assembly is regulated by distinct modes of Rho signaling in yeast. J Cell Biol 161: 1081-92.

17 Evangelista M, Blundell K, Longtine MS, Chow CJ, et al. 1997. Bni1p, a yeast formin linking cdc42p and the actin cytoskeleton during polarized morphogenesis. Science 276: 118-22.

18 Martin SG, Rincon SA, Basu R, Perez P, et al. 2007. Regulation of the formin for3p by cdc42p and bud6p. Mol Biol Cell 18: 4155-67.

19 Wu H, Turner C, Gardner J, Temple B, et al. 2010. The exo70 subunit of the exocyst is an effector for both cdc42 and rho3 function in polarized exocytosis. Mol Biol Cell 21: 430-42.

20 Zhang X, Bi E, Novick P, Du L, et al. 2001. Cdc42 interacts with the exocyst and regulates polarized secretion. J Biol Chem 276: 46745-50.

21 Zhang X, Orlando K, He B, Xi F, et al. 2008. Membrane association and functional regulation of Sec3 by phospholipids and Cdc42.J Cell Biol 180: 145-58.

22 Bendezu F0, Martin SG. 2011. Actin cables and the exocyst form two independent morphogenesis pathways in the fission yeast. Mol Biol Cell 22: 44-53.

23 Turing AM. 1952. The Chemical Basis of Morphogenesis. Philosophical Transactions of the Royal Society of London Series B 237: 37-72.

24 Goryachev AB, Pokhilko AV. 2008. Dynamics of Cdc42 network embodies a Turing-type mechanism of yeast cell polarity. FEBS Lett 582: 1437-43.

25 Irazoqui JE, Gladfelter AS, Lew DJ. 2003. Scaffold-mediated symmetry breaking by Cdc42p. Nat Cell Biol 5: 1062-70.

26 Wedlich-Soldner R, Altschuler S, Wu L, Li R. 2003. Spontaneous cell polarization through actomyosin-based delivery of the Cdc42 GTPase. Science 299: 1231-5.

27 Mogilner A, Allard J, Wollman R. 2012. Cell polarity: quantitative modeling as a tool in cell biology. Science 336: 175-9.

28 Butty AC, Perrinjaquet N, Petit A, Jaquenoud M, et al. 2002. A positive feedback loop stabilizes the guanine-nucleotide exchange factor Cdc24 at sites of polarization. Embo J 21: 1565-76.

29 Kozubowski L, Saito K, Johnson JM, Howell AS, et al. 2008. Symmetrybreaking polarization driven by a Cdc42p GEF-PAK complex. Curr Biol 18: 1719-26.

30 Wedlich-Soldner R, Wai SC, Schmidt T, Li R. 2004. Robust cell polarity is a dynamic state established by coupling transport and GTPase signaling. J Cell Biol 166: 889-900.

31 Endo M, Shirouzu M, Yokoyama S. 2003. The Cdc42 binding and scaffolding activities of the fission yeast adaptor protein Scd2.J Biol Chem 278: 843-52. 
32 Chang EC, Barr M, Wang Y, Jung V, et al. 1994. Cooperative interaction of $\mathrm{S}$. pombe proteins required for mating and morphogenesis. Cell 79: 131-41.

33 Wai SC, Gerber SA, Li R. 2009. Multisite phosphorylation of the guanine nucleotide exchange factor Cdc24 during yeast cell polarization. PLoS One 4: e6563.

34 Bose I, Irazoqui JE, Moskow JJ, Bardes ES, et al. 2001. Assembly of scaffold-mediated complexes containing Cdc42p, the exchange factor Cdc24p, and the effector Cla4p required for cell cycle-regulated phosphorylation of Cdc24p. J Biol Chem 276: 7176-86.

35 Gulli MP, Jaquenoud M, Shimada Y, Niederhauser G, et al. 2000. Phosphorylation of the Cdc42 exchange factor Cdc24 by the PAK-like kinase Cla4 may regulate polarized growth in yeast. Mol Cell 6: 1155-67.

36 Kuo CC, Savage NS, Chen H, Wu CF, et al. 2014. Inhibitory GEF phosphorylation provides negative feedback in the yeast polarity circuit. Curr Biol 24: 753-9.

37 France YE, Boyd C, Coleman J, Novick PJ. 2006. The polarityestablishment component Bem1p interacts with the exocyst complex through the Sec15p subunit. J Cell Sci 119: 876-88.

38 Liu D, Novick P. 2014. Bem1p contributes to secretory pathway polarization through a direct interaction with Exo70p. J Cell Biol 207: 5972.

39 Bähler J, Peter M. 2000. Cell polarity in yeast. In Drubin D, ed; Cell Polarity: Oxford University Press. p 21-77.

40 Park HO, Bi E. 2007. Central roles of small GTPases in the development of cell polarity in yeast and beyond. Microbiol Mol Biol Rev 71: 48-96.

41 Chant J, Herskowitz I. 1991. Genetic control of bud site selection in yeast by a set of gene products that constitute a morphogenetic pathway. Cell 65: 1203-12.

42 Bi E, Park HO. 2012. Cell polarization and cytokinesis in budding yeast. Genetics 191: 347-87.

43 Smith SE, Rubinstein B, Mendes Pinto I, Slaughter BD, et al. 2013. Independence of symmetry breaking on Bem1-mediated autocatalytic activation of Cdc42. J Cell Biol 202: 1091-106.

44 Shimada Y, Wiget P, Gulli MP, Bi E, et al. 2004. The nucleotide exchange factor Cdc24p may be regulated by auto-inhibition. Embo J 23: 1051-62.

45 Park HO, Bi E, Pringle JR, Herskowitz I. 1997. Two active states of the Ras-related Bud1/Rsr1 protein bind to different effectors to determine yeast cell polarity. Proc Natl Acad Sci U S A 94: 4463-8.

46 Zheng Y, Bender A, Cerione RA. 1995. Interactions among proteins involved in bud-site selection and bud-site assembly in Saccharomyces cerevisiae. J Biol Chem 270: 626-30.

47 Li R, Wedlich-Soldner R. 2009. Bem1 complexes and the complexity of yeast cell polarization. Curr Biol 19: R194-5; author reply R5.

48 Kozminski KG, Beven L, Angerman E, Tong AH, et al. 2003. Interaction between a Ras and a Rho GTPase couples selection of a growth site to the development of cell polarity in yeast. Mol Biol Cell 14: 4958-70. 
49 Kang PJ, Beven L, Hariharan S, Park Ho. 2010. The Rsr1/Bud1 GTPase interacts with itself and the Cdc42 GTPase during bud-site selection and polarity establishment in budding yeast. Mol Biol Cell 21: 3007-16.

50 Kelly FD, Nurse P. 2011. De novo growth zone formation from fission yeast spheroplasts. PLoS One 6: e27977.

51 Kelly FD, Nurse P. 2011. Spatial control of Cdc42 activation determines cell width in fission yeast. Mol Biol Cell 22: 3801-11.

52 Das M, Wiley DJ, Chen X, Shah K, et al. 2009. The conserved NDR kinase Orb6 controls polarized cell growth by spatial regulation of the small GTPase Cdc42. Curr Biol 19: 1314-9.

53 Kokkoris K, Castro DG, Martin SG. 2014. Tea4-phosphatase I landmark promotes local growth by dual Cdc42 GEF recruitment and GAP exclusion. J Cell Sci.

54 Vjestica A, Zhang D, Liu J, Oliferenko S. 2013. Hsp70-Hsp40 chaperone complex functions in controlling polarized growth by repressing Hsf1driven heat stress-associated transcription. PLoS genetics 9: e1003886.

55 Coll PM, Trillo Y, Ametzazurra A, Perez P. 2003. Gef1p, a New Guanine Nucleotide Exchange Factor for Cdc42p, Regulates Polarity in Schizosaccharomyces pombe. Mol Biol Cell 14: 313-23.

56 Hirota K, Tanaka K, Ohta K, Yamamoto M. 2003. Gef1p and Scd1p, the Two GDP-GTP exchange factors for Cdc42p, form a ring structure that shrinks during cytokinesis in Schizosaccharomyces pombe. Mol Biol Cell 14: 3617-27.

57 Kang PJ, Lee ME, Park HO. 2014. Bud3 activates Cdc42 to establish a proper growth site in budding yeast. J Cell Biol 206: 19-28.

58 Lee ME, Lo WC, Miller KE, Chou CS, et al. 2015. Regulation of Cdc42 polarization by the Rsr1 GTPase and Rga1, a Cdc42 GTPase activating protein, in budding yeast. J Cell Sci.

59 Caviston JP, Tcheperegine SE, Bi E. 2002. Singularity in budding: a role for the evolutionarily conserved small GTPase Cdc42p. Proc Natl Acad Sci US A 99: 12185-90.

60 Bendezu FO, Vincenzetti V, Vavylonis D, Wyss R, et al. 2015. Spontaneous Cdc42 polarization independent of GDI-mediated extraction and actin-based trafficking. PLoS Biol 13: e1002097.

61 Freisinger T, Klunder B, Johnson J, Muller N, et al. 2013. Establishment of a robust single axis of cell polarity by coupling multiple positive feedback loops. Nat Commun 4: 1807.

62 Tiedje C, Sakwa I, Just U, Hofken T. 2008. The Rho GDI Rdi1 regulates Rho GTPases by distinct mechanisms. Mol Biol Cell 19: 2885-96.

63 Nakano K, Mutoh T, Arai R, Mabuchi I. 2003. The small GTPase Rho4 is involved in controlling cell morphology and septation in fission yeast. Genes Cells 8: 357-70.

64 Slaughter BD, Das A, Schwartz JW, Rubinstein B, et al. 2009. Dual modes of cdc42 recycling fine-tune polarized morphogenesis. Dev Cell 17: 823-35.

65 Orlando K, Sun X, Zhang J, Lu T, et al. 2011. Exo-endocytic trafficking and the septin-based diffusion barrier are required for the maintenance of Cdc42p polarization during budding yeast asymmetric growth. Mol Biol Cell 22: 624-33. 

polarization: rewiring yeast cells to make two buds. Cell 139: 731-43.

67 Savage NS, Layton AT, Lew DJ. 2012. Mechanistic mathematical model of polarity in yeast. Mol Biol Cell 23: 1998-2013.

68 Layton AT, Savage NS, Howell AS, Carroll SY, et al. 2011. Modeling vesicle traffic reveals unexpected consequences for Cdc42p-mediated polarity establishment. Curr Biol 21: 184-94.

69 Watson LJ, Rossi G, Brennwald P. 2014. Quantitative analysis of membrane trafficking in regulation of Cdc42 polarity. Traffic 15: 1330-43.

70 Slaughter BD, Unruh JR, Das A, Smith SE, et al. 2013. Non-uniform membrane diffusion enables steady-state cell polarization via vesicular trafficking. Nat Commun 4.

71 Valdez-Taubas J, Pelham HR. 2003. Slow diffusion of proteins in the yeast plasma membrane allows polarity to be maintained by endocytic cycling. Curr Biol 13: 1636-40.

72 Marco E, Wedlich-Soldner R, Li R, Altschuler SJ, et al. 2007. Endocytosis optimizes the dynamic localization of membrane proteins that regulate cortical polarity. Cell 129: 411-22.

73 Howell AS, Jin M, Wu CF, Zyla TR, et al. 2012. Negative feedback enhances robustness in the yeast polarity establishment circuit. Cell 149: 322-33.

74 Fairn GD, Hermansson M, Somerharju P, Grinstein S. 2011. Phosphatidylserine is polarized and required for proper $\mathrm{Cdc} 42$ localization and for development of cell polarity. Nat Cell Biol 13: 142430.

75 Das A, Slaughter BD, Unruh JR, Bradford WD, et al. 2012. Flippasemediated phospholipid asymmetry promotes fast Cdc42 recycling in dynamic maintenance of cell polarity. Nat Cell Biol 14: 304-10.

76 Okada S, Leda M, Hanna J, Savage NS, et al. 2013. Daughter cell identity emerges from the interplay of Cdc42, septins, and exocytosis. Dev Cell 26: 148-61.

77 Dyer JM, Savage NS, Jin M, Zyla TR, et al. 2012. Tracking Shallow Chemical Gradients by Actin-Driven Wandering of the Polarization Site. Curr Biol.

78 Irazoqui JE, Howell AS, Theesfeld CL, Lew DJ. 2005. Opposing roles for actin in Cdc42p polarization. Mol Biol Cell 16: 1296-304.

79 Qin Y, Yang Z. 2011. Rapid tip growth: insights from pollen tubes. Semin Cell Dev Biol 22: 816-24.

80 Wu CF, Lew DJ. 2013. Beyond symmetry-breaking: competition and negative feedback in GTPase regulation. Trends Cell Biol 23: 476-83.

81 Bendezu Fo, Martin SG. 2012. Cdc42 oscillations in yeasts. Science signaling 5: pe53.

82 Altschuler SJ, Angenent SB, Wang Y, Wu LF. 2008. On the spontaneous emergence of cell polarity. Nature 454: 886-9.

83 Das M, Drake T, Wiley DJ, Buchwald P, et al. 2012. Oscillatory Dynamics of Cdc42 GTPase in the Control of Polarized Growth. Science.

84 Ozbudak EM, Becskei A, van Oudenaarden A. 2005. A system of counteracting feedback loops regulates Cdc42p activity during spontaneous cell polarization. Dev Cell 9: 565-71. 
85 Bendezu F0, Martin SG. 2013. Cdc42 Explores the Cell Periphery for Mate Selection in Fission Yeast. Curr Biol 23: $42-7$.

86 Bonazzi D, Julien JD, Romao M, Seddiki R, et al. 2014. Symmetry Breaking in Spore Germination Relies on an Interplay between Polar Cap Stability and Spore Wall Mechanics. Dev Cell 28: 534-46.

87 Wu CF, Savage NS, Lew DJ. 2013. Interaction between bud-site selection and polarity-establishment machineries in budding yeast. Philos Trans $R$ Soc Lond B Biol Sci 368: 20130006.

88 Klunder B, Freisinger T, Wedlich-Soldner R, Frey E. 2013. GDImediated cell polarization in yeast provides precise spatial and temporal control of Cdc42 signaling. PLoS Comput Biol 9: e1003396.

89 Das M, Wiley DJ, Medina S, Vincent HA, et al. 2007. Regulation of cell diameter, For3p localization, and cell symmetry by fission yeast Rho-GAP Rga4p. Mol Biol Cell 18: 2090-101.

90 Tatebe H, Nakano K, Maximo R, Shiozaki K. 2008. Pom1 DYRK regulates localization of the Rga4 GAP to ensure bipolar activation of Cdc42 in fission yeast. Curr Biol 18: 322-30.

91 Tong Z, Gao XD, Howell AS, Bose I, et al. 2007. Adjacent positioning of cellular structures enabled by a Cdc42 GTPase-activating proteinmediated zone of inhibition. J Cell Biol 179: 1375-84.

92 Knaus M, Pelli-Gulli MP, van Drogen F, Springer S, et al. 2007. Phosphorylation of Bem2p and Bem3p may contribute to local activation of Cdc42p at bud emergence. EMBO J 26: 4501-13.

93 Feierbach B, Chang F. 2001. Roles of the fission yeast formin for3p in cell polarity, actin cable formation and symmetric cell division. Curr Biol 11: 1656-65.

94 Rupes I, Jia Z, Young PG. 1999. Ssp1 promotes actin depolymerization and is involved in stress response and new end take-off control in fission yeast. Mol Biol Cell 10: 1495-510.

95 Martin SG, Chang F. 2005. New end take off: regulating cell polarity during the fission yeast cell cycle. Cell Cycle 4: 1046-9.

96 Martin SG, McDonald WH, Yates JR, 3rd, Chang F. 2005. Tea4p links microtubule plus ends with the formin for3p in the establishment of cell polarity. Dev Cell 8: 479-91.

97 Mata J, Nurse P. 1997. tea1 and the microtubular cytoskeleton are important for generating global spatial order within the fission yeast cell. Cell 89: 939-49.

98 Tatebe H, Shimada K, Uzawa S, Morigasaki S, et al. 2005. Wsh3/Tea4 is a novel cell-end factor essential for bipolar distribution of Tea1 and protects cell polarity under environmental stress in S. pombe. Curr Biol 15: 1006-15.

99 Minc N, Bratman SV, Basu R, Chang F. 2009. Establishing new sites of polarization by microtubules. Curr Biol 19: 83-94.

100 Terenna CR, Makushok T, Velve-Casquillas G, Baigl D, et al. 2008. Physical mechanisms redirecting cell polarity and cell shape in fission yeast. Curr Biol 18: 1748-53. 
Figure legends

\section{Figure 1: The Cdc42 activation cycle}

The small GTPase Cdc42 is inserted at the membrane by prenylation. When GTPbound, Cdc42 activates effector proteins, such as PAK-family kinases, actin nucleators and the exocyst, which lead to effective cell polarization. Cdc42 cycles between its active and inactive, GDP-bound form. Cdc42 activation is catalysed by Guanine nucleotide Exchange Factors (GEFs), which lead to exchange of the GDP for a new GTP. GTP hydrolysis results both from Cdc42's intrinsic GTPase activity and GTPase Activating Proteins (GAPs). Cdc42 can also be extracted from the membrane, preferentially in the GDP-bound form, by associating with the Guanine nucleotide Dissociation Inhibitor (GDI), which masks the prenyl group and solubilizes Cdc42 in the cytosol. In proliferating cells, Cdc42 is active at the presumptive bud site in $S$. cerevisiae (left) and at the two cell poles in bipolar $S$. pombe cells (right).

\section{Figure 2: Self-amplification of Cdc42 activity}

Demonstrated and putative positive feedback amplification mechanisms of Cdc42 activity are shown. The Bem1-dependent PAK-GEF complex is shown in green. This feedback system is essential in absence of Rsr1 in S. cerevisiae. The putative Ras-dependent positive feedback is shown in purple. This feedback system is essential in absence of landmarks and the Bem 1 system. However, how Cdc42 activity may positively enhance Ras activation is not known. For Ras and the Cdc42 GEF in these two systems, generic names are shown, as well as, in this order, specific $S$. cerevisiae and $S$. pombe names. A second Cdc42 GEF exists in both $S$. cerevisiae (Bud3) and S. pombe (Gef1). Though these GEFs are not homologs, they may both underlie additional putative feedback systems. Dashed arrows and question marks highlight the putative regulatory steps.

\section{Figure 3: Local enrichment of Cdc42 as a result of the slower dynamics of Cdc42-GTP vs. Cdc42-GDP}

The schematic illustrates how the observed faster dynamics of Cdc42-GDP, both as GDI substrate for membrane extraction and through lateral diffusion at the plasma membrane, relative to Cdc42-GTP may underlie the local accumulation of Cdc42 at sites of activity. Positive feedback on Cdc42 activation is represented by the red arrow enhancing the conversion of Cdc42-GDP to Cdc42-GTP. Cdc42-GDP can be extracted from the membrane and solubilized in the cytosol by the GDI. Cdc42-GDP also diffuses faster at the membrane than Cdc42-GTP, represented by the bidirectional arrows along the membrane. As the polar cap grows, the fast dynamics of Cdc42-GDP provide a net influx of Cdc42 into the cap. At steady state, this influx is balanced by Cdc42-GTP hydrolysis. 
Figure 4: F-actin and membrane flux contribute to stabilization of the polar cap

A. The schematic illustrates the effect of actin depolymerization (right) on the stability and location of the polar cap in S. cerevisiae (top) and S. pombe (bottom) proliferating cells. B. Exo- and endocytic trafficking underlies the membrane flux at the polar cap. This leads to lipid as well as protein fluxes. Actin patchesmediated endocytosis destabilizes the polar cap (-), while actin cable-mediated exocytic vesicle transport antagonizes this effect and provides a net benefit to the polar cap (+). Exocytosis also contributes to polar cap stabilization and morphogenesis in $S$. cerevisiae by displacing septins to a peripheral ring (bottom). 
Figure 1

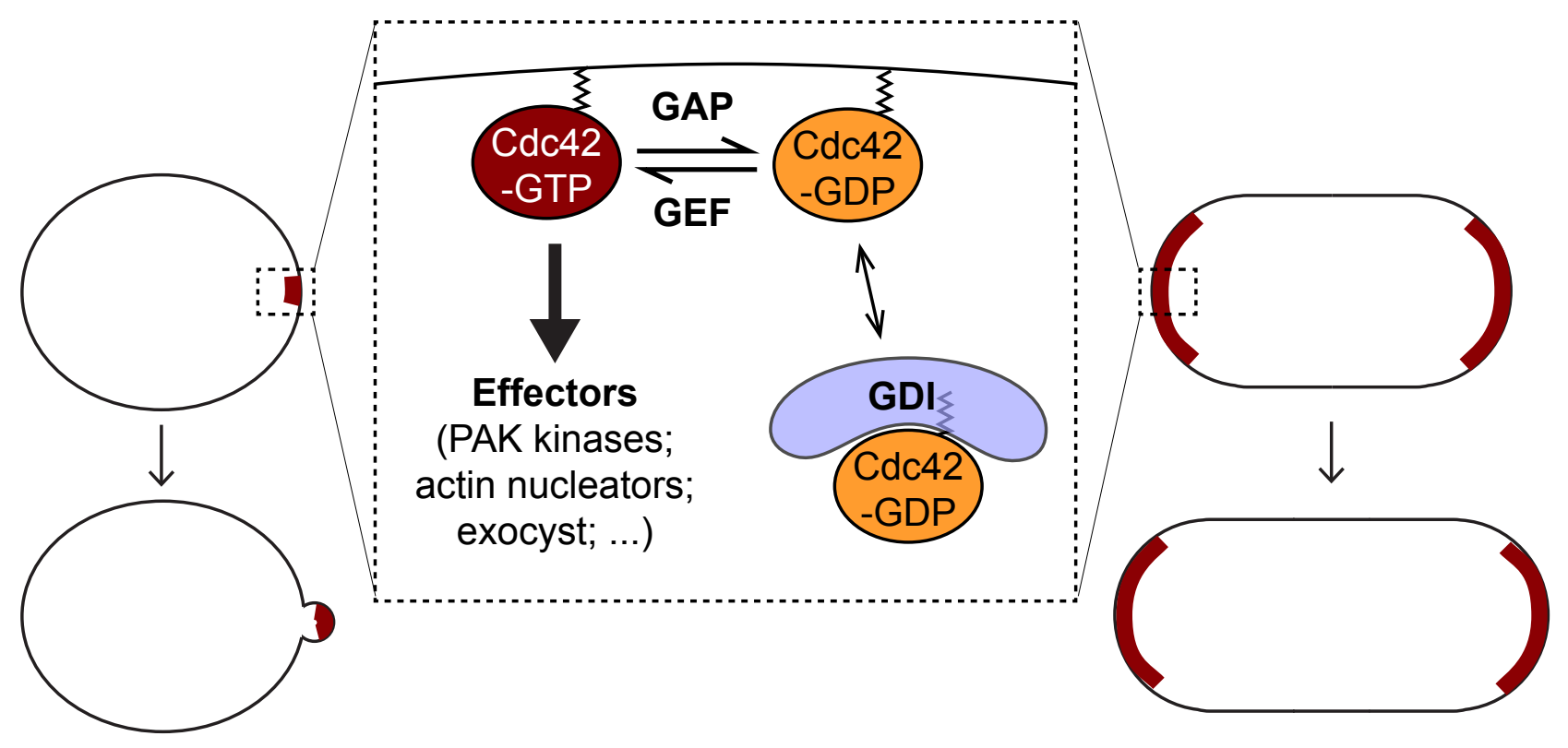


Figure 2

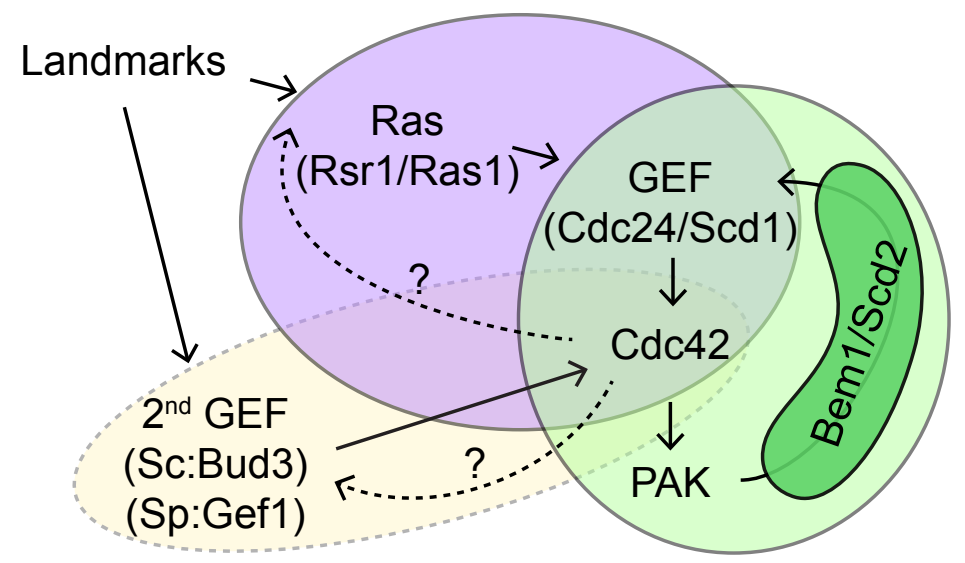


Figure 3

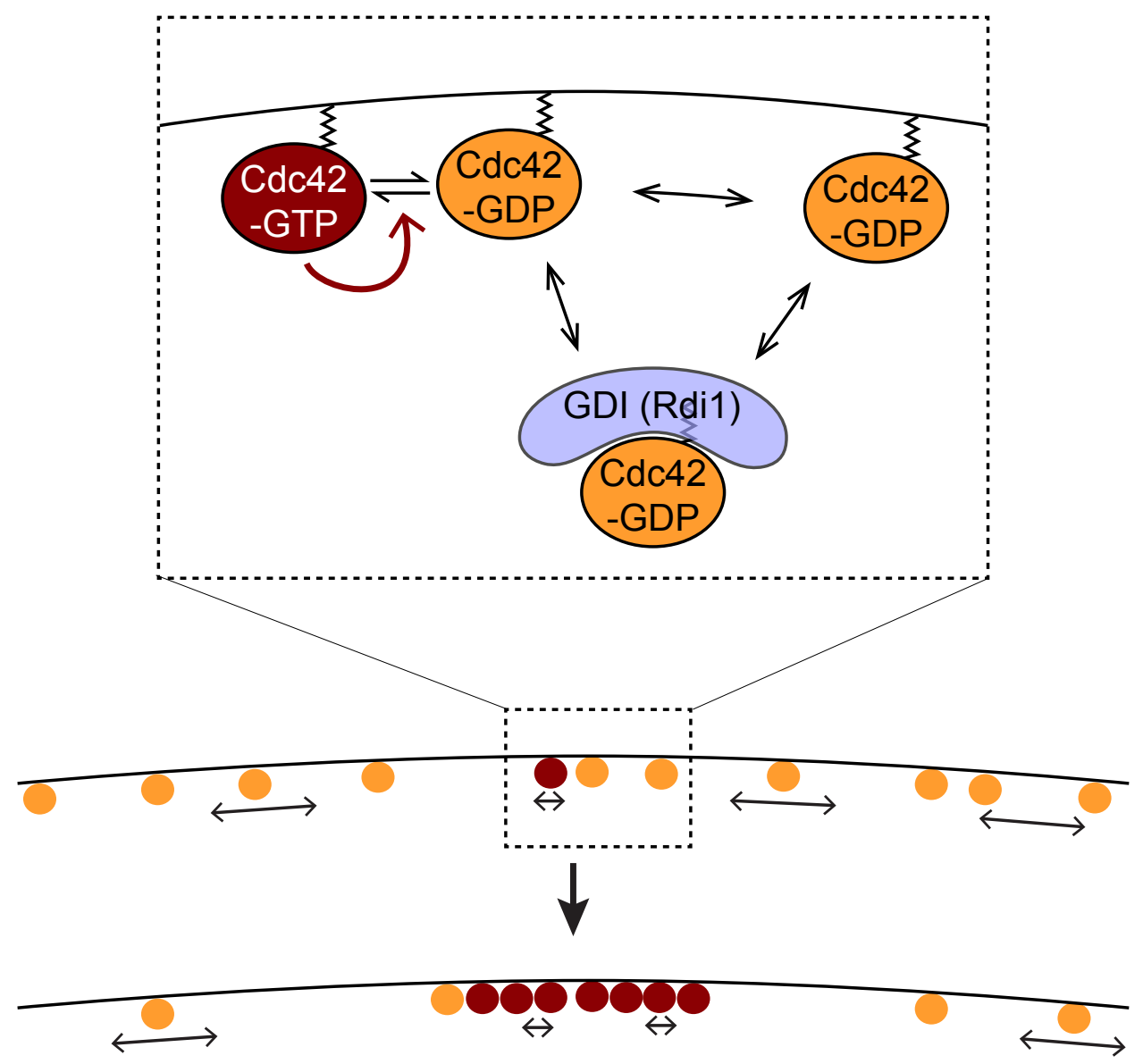


Figure 4

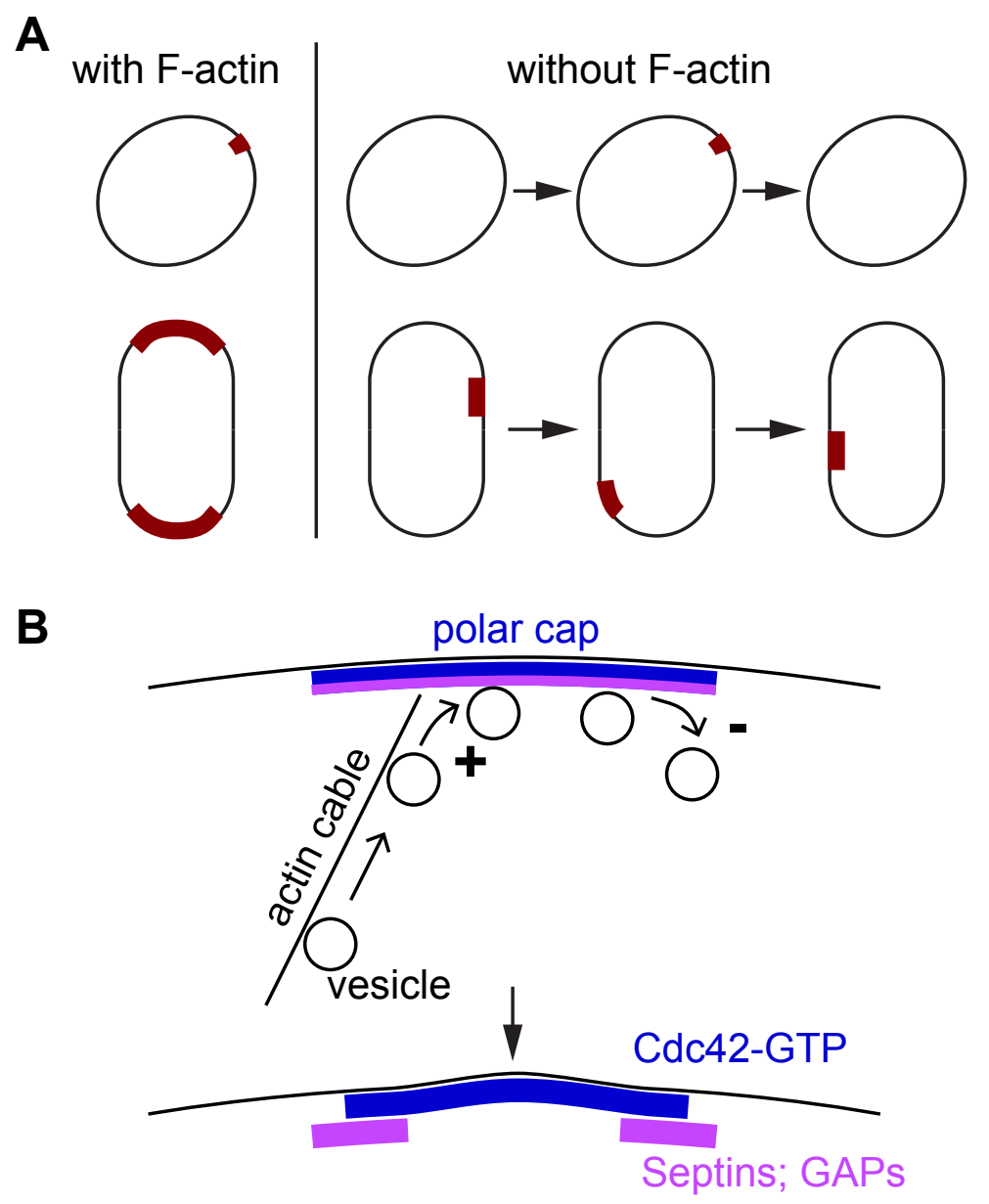

